\title{
Search for Heavy Neutral Higgs Bosons at the LHC
}

\author{
M. Ashry ${ }^{1}, 1,2$ K. Ezzat, ${ }^{2,3}$ and S. Khalii ${ }^{2}$ \\ ${ }^{1}$ Department of Mathematics, Faculty of Science, Cairo University, Giza 12613, Egypt \\ ${ }^{2}$ Center for Fundamental Physics, Zewail City of Science and Technology, 6th of October City, Giza 12578, Egypt \\ ${ }^{3}$ Department of Mathematics, Faculty of Science, Ain Shams University, Cairo 11566, Egypt
}

\begin{abstract}
$\overline{\text { Abstract }}$
A search for a CP-even heavy Higgs boson at the LHC is analysed in a Left-Right model with minimal Higgs sector. We report our results for potential signatures for heavy neutral Higgs decay into two SMlike Higgs bosons, namely $h^{\prime} \rightarrow h h \rightarrow b \bar{b} \gamma \gamma$ for a center-of-mass energy $\sqrt{s}=14 \mathrm{TeV}$ and integrated luminosity $L_{\mathrm{int}}=300,3000 \mathrm{fb}^{-1}$.
\end{abstract}

Keywords: beyond the standard model, left-right symmetry and Higgs phenomenology

DOI: 10.31526/ACP.BSM-2021.21

We recently investigated a Left-Right model with minimal Higgs sector, based on the gauge group $S U(3)_{C} \times S U(2)_{L} \times S U(2)_{R} \times$ $U(1)_{B-L}$ as in other conventional left-right models [1,2]. Only one scalar bidoublet and a scalar right-handed doublet were considered [3]. We demonstrated that the tiny values of light neutrino masses in this model are generated by an inverse-seesaw mechanism $[4,5,6,7,8]$. We also investigated LHC searches for the CP-even heavy Higgs boson. In this article, we summarize our findings for the signature of heavy neutral Higgs decay to two SM-like Higgs bosons, namely $h^{\prime} \rightarrow h h \rightarrow b \bar{b} \gamma \gamma$ at integrated luminosity $L_{\text {int }}=300,3000 \mathrm{fb}^{-1}[9,10]$.

In our model of Left-Right with Inverse Seesaw (LRIS), the general Higgs potential is given by [11]

$$
\begin{aligned}
V\left(\phi, \chi_{R}\right) & =\mu_{1} \operatorname{Tr}\left(\phi^{\dagger} \phi\right)+\mu_{2}\left[\operatorname{Tr}\left(\tilde{\phi} \phi^{\dagger}\right)+\operatorname{Tr}\left(\tilde{\phi}^{\dagger} \phi\right)\right]+\lambda_{1}\left(\operatorname{Tr}\left(\phi^{\dagger} \phi\right)\right)^{2}+\lambda_{2}\left[\left(\operatorname{Tr}\left(\tilde{\phi} \phi^{\dagger}\right)\right)^{2}+\left(\operatorname{Tr}\left(\tilde{\phi}^{\dagger} \phi\right)\right)^{2}\right] \\
& +\lambda_{3} \operatorname{Tr}\left(\tilde{\phi} \phi^{\dagger}\right) \operatorname{Tr}\left(\tilde{\phi}^{\dagger} \phi\right)+\lambda_{4} \operatorname{Tr}\left(\phi \phi^{\dagger}\right)\left(\operatorname{Tr}\left(\tilde{\phi} \phi^{\dagger}\right)+\operatorname{Tr}\left(\tilde{\phi}^{\dagger} \phi\right)\right)+\mu_{3}\left(\chi_{R}^{\dagger} \chi_{R}\right)+\rho_{1}\left(\chi_{R}^{\dagger} \chi_{R}\right)^{2} \\
& +\alpha_{1} \operatorname{Tr}\left(\phi^{\dagger} \phi\right)\left(\chi_{R}^{\dagger} \chi_{R}\right)+\alpha_{2}\left(\chi_{R}^{\dagger} \phi^{\dagger} \phi \chi_{R}\right)+\alpha_{3}\left(\chi_{R}^{\dagger} \tilde{\phi}^{\dagger} \tilde{\phi} \chi_{R}\right)+\alpha_{4}\left(\chi_{R}^{\dagger} \phi^{\dagger} \tilde{\phi} \chi_{R}+\text { h.c. }\right) .
\end{aligned}
$$

The constraints imposed on the above potential parameters due the minimization conditions and the boundedness from below are provided in Appendix of Ref. [3]. After Left-Right and Electroweak symmetry breaking, the following CP-even neutral Higgs mass matrix is obtained:

$$
\left(M_{H}^{2}\right)_{i j}=\left.\frac{\partial^{2} V\left(\phi, \chi_{L, R}\right)}{\partial \phi_{i}^{0 R} \partial \phi_{j}^{0 R}}\right|_{\left\langle\phi_{i / j}^{0 R}\right\rangle=v_{i / j},\left\langle\phi_{i / j}^{0 I}\right\rangle=0},
$$

where $i / j \equiv 1,2$ and $R$ refer for neutral components: $\phi_{1}$ and $\phi_{2}$ of the bidoublet and neutral component of $\chi_{R}$. In this case, one find the following matrix elements:

$$
\begin{aligned}
& m_{11}=2 v^{2}\left(\lambda_{1} s_{\beta}^{2}+\lambda_{23} c_{\beta}^{2}+\lambda_{4} s_{2 \beta}\right)+\frac{1}{4}\left(\frac{1}{c_{2 \beta}}+1\right) \alpha_{32} v_{R}^{2}, \\
& m_{12}=m_{21}=v^{2}\left(\left(\lambda_{1}+\lambda_{23}\right) s_{2 \beta}+2 \lambda_{4}\right)-\frac{1}{4} \alpha_{32} v_{R}^{2} t_{2 \beta}, \\
& m_{13}=m_{31}=v v_{R}\left(\alpha_{13} s_{\beta}+\alpha_{4} c_{\beta}\right), \\
& m_{22}=2 v^{2}\left(\lambda_{1} c_{\beta}^{2}+\lambda_{23} s_{\beta}^{2}+\lambda_{4} s_{2 \beta}\right)+\frac{1}{4}\left(\frac{1}{c_{2 \beta}}-1\right) \alpha_{32} v_{R}^{2}, \\
& m_{23}=m_{32}=v v_{R}\left(\alpha_{12} c_{\beta}+\alpha_{4} s_{\beta}\right), \\
& m_{33}=2 \rho_{1} v_{R}^{2},
\end{aligned}
$$

where $\alpha_{1 i}=\alpha_{1}+\alpha_{i}, i=2,3$ and $\lambda_{23}=2 \lambda_{2}+\lambda_{3}$. This matrix can be diagonalized by a unitary transformation matrix $Z^{H}$ such that $\left(\phi_{1}^{0 R}, \phi_{2}^{0 R}, \chi_{R}^{0 R}\right)^{T}=Z^{H T}\left(H_{1}, H_{2}, H_{3}\right)^{T}$. The coefficients of the rotation matrix $Z^{H}$ are given explicitly in Ref. [3]. We identify the

\footnotetext{
${ }^{1}$ Speaker
} 
lightest eigenstate $H_{1} \equiv h$ to be the SM-like Higgs boson that we fix its mass with $m_{h}=125 \mathrm{GeV}[12,13]$. The other two eigenvalues are given by

$$
m_{H_{2,3}}^{2}=\frac{1}{2}\left(T^{h}-m_{h}^{2} \mp \sqrt{\left(T^{h}-m_{h}^{2}\right)^{2}-\frac{4 D^{h}}{m_{h}^{2}}}\right),
$$

where $T^{h}$ and $D^{h}$ are the trace and determinant of $M_{H}^{2}$. From Eq. (9), one can show that the next lightest $C P$-even neutral Higgs boson, $H_{2} \equiv h^{\prime}$, could have a mass of order a few hundred GeVs. In our analysis we vary the relevant parameters in the scalar potential in the following ranges

$$
\lambda_{1} \in[0.18,0.30], \quad \lambda_{4} \in[0.70,0.99], \quad \alpha_{1} \in[0.06,0.16], \quad \alpha_{4} \in[0.60,0.99], \quad \rho_{1} \in[0.08,0.14], \quad \lambda_{23} \in[-0.1,3] .
$$

The physical eigenstate $h^{\prime}$ (second lightest neutral Higgs boson) can be written in terms of components of real parts of neutral scalar doublets as follows:

$$
h^{\prime}=Z_{21}^{H} \phi_{1}^{0 R}+Z_{22}^{H} \phi_{2}^{0 R}+Z_{23}^{H} \chi_{R}^{0 R} .
$$

We chose the three benchmark points, as emphasized in [3] with $m_{h^{\prime}}=250,400$ and $600 \mathrm{GeV}$. We have shown that $h^{\prime}$ is essentially formed from $\phi_{1}$ component with very smaller contributions from $\phi_{2}$ and $\chi_{R}$ components.

The relevant coupling of $h^{\prime}$ with the SM-like Higgs boson, $g_{h^{\prime}} h h$ is given by

$$
g_{h^{\prime} h h} \approx-2 i Z_{21}^{H} Z_{12}^{H}\left\{v\left(\left(\lambda_{1}-\lambda_{23}\right) c_{\beta}+3 \lambda_{4} s_{\beta}\right) Z_{12}^{H}+\alpha_{4} v_{R} Z_{13}^{H}\right\},
$$

where $Z^{H}$ is the CP-even neutral Higgs bosons mixing matrix. Here, we fix the gauge couplings as follows: $g_{L}=g_{R}=g_{2}=$ 0.663, $g_{B L}=0.422$, and $v_{R}=6400 \mathrm{GeV}$ and the potential parameters $\alpha_{i}, \lambda_{i}$ and $\rho_{1}$ are varied within mentioned ranges in Eq. (10).

The heavy Higgs boson, $h^{\prime}$, is mainly produced at the LHC from gluon-gluon fusion (ggF) process, which induces about $90 \%$ of its total production cross section at the LHC. In Fig. 1 (left) we show the $h^{\prime}$ ggF-production cross section versus $m_{h^{\prime}}$. note that from this figure the $\sigma\left(p p \rightarrow h^{\prime}\right)$ can be of order $\gtrsim 2 \mathrm{pb}$ for $m_{h^{\prime}} \lesssim 400 \mathrm{GeV}$. so we consider $L_{\mathrm{int}}=300 \mathrm{fb}^{-1} \mathrm{for}$ light $h^{\prime}$ and $L_{\text {int }}=3000 \mathrm{fb}^{-1}$ for heavy, at $\sqrt{s}=14 \mathrm{TeV}$.
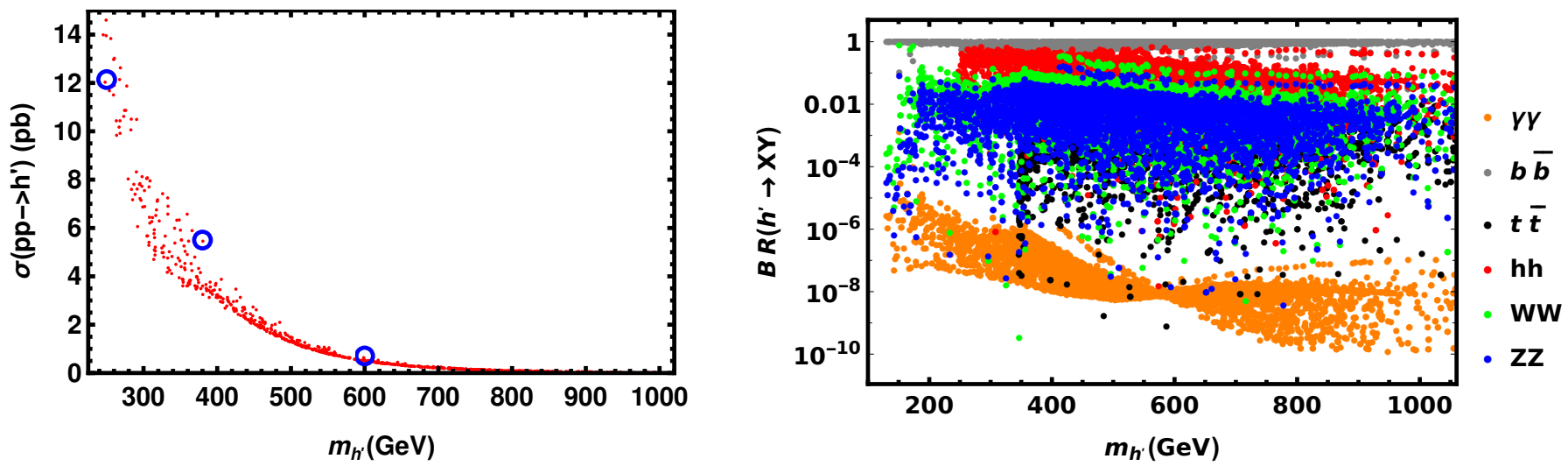

FIGURE 1: (Left) The $h^{\prime}$ production cross section from ggF as a function of its mass $m_{h^{\prime}}$. The three above mentioned benchmark points are surrounded by blue circles. (Right) Branching ratios (BR) of $h^{\prime}$ decays versus its mass $m_{h^{\prime}}$. The scan on the relevant parameters are considered as explained above.

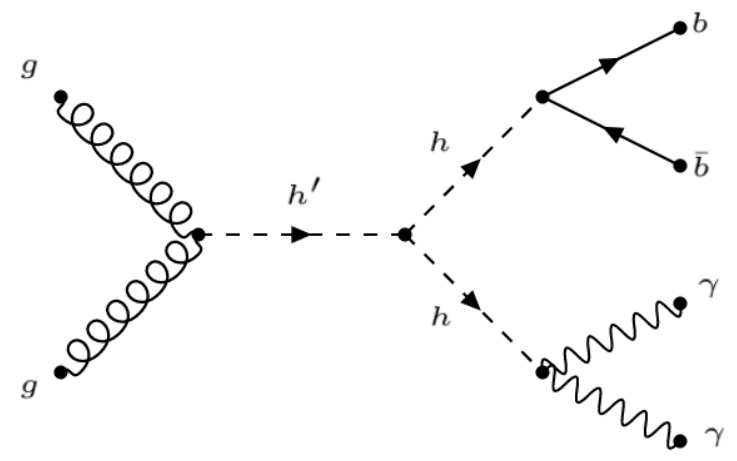

FIGURE 2: Feynman diagram for the $h^{\prime}$ ggF production and decay process $g g \rightarrow h^{\prime} \rightarrow h h \rightarrow b \bar{b} \gamma \gamma$ 
In Fig. 1 (right) we show the relevant $h^{\prime}$ decay branching ratios as functions of $m_{h^{\prime}}$. It is remarkable to notice that for $m_{h^{\prime}} \leq$ $600 \mathrm{GeV}$, the $h^{\prime}$ decay branching ratio to two SM Higgs boson is not small, mainly $\mathrm{BR}\left(h^{\prime} \rightarrow h h\right) \geq 10 \%$, which gives a hope for probing this heavy Higgs through this channel.

we have alot chance for probe $h^{\prime}$ like $h^{\prime} \rightarrow h h \rightarrow b \bar{b} b \bar{b}$ but this this process has a huge background also can go to process $h^{\prime} \rightarrow h h \rightarrow 2 b+2 W \rightarrow$ bb $\ell \ell \ell v$ but this has very small cross section, so we will start with a good process for probing $h^{\prime}$ is $h^{\prime} \rightarrow h h \rightarrow b \bar{b} \gamma \gamma$ see the Feynman diagram in Fig. 2. we make analysis wuth three masses for $h^{\prime} m_{h^{\prime}}=250 \mathrm{GeV}, 400 \mathrm{GeV}$ and $600 \mathrm{GeV}$.
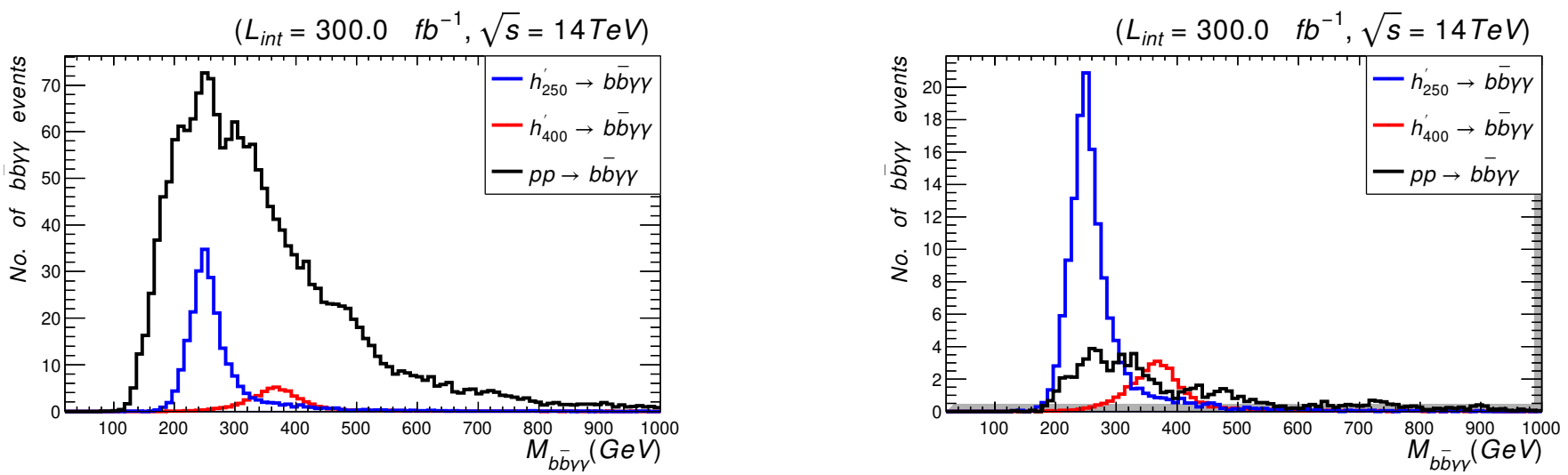

FIGURE 3: Number of signal events for $h^{\prime} \rightarrow b \bar{b} \gamma \gamma$ decays at mass $m_{h^{\prime}}=250 \mathrm{GeV}$ (blue) and $400 \mathrm{GeV}$ (red) induced by ggF versus the invariant mass of the final states $b \bar{b} \gamma \gamma$, at $\sqrt{s}=14 \mathrm{TeV}$ and $L_{\mathrm{int}}=300 \mathrm{fb}^{-1}$ alongside the relevant background events (black) before (left) and after (right) applying the cut flow of Tab. 2. The corresponding vlaues of cross sections and branching ratios are given in Tab. 1.

by use the narrow width approximation we can calculate the total cross section $\sigma\left(p p \rightarrow h^{\prime} \rightarrow h h \rightarrow b \bar{b} \gamma \gamma\right)$ as

$$
\sigma\left(p p \rightarrow h^{\prime} \rightarrow h h \rightarrow b \bar{b} \gamma \gamma\right) \approx \sigma\left(p p \rightarrow h^{\prime}\right) \times \mathrm{BR}\left(h^{\prime} \rightarrow h h\right) \times \mathrm{BR}(h \rightarrow b \bar{b}) \times \mathrm{BR}(h \rightarrow \gamma \gamma)
$$

where the $h^{\prime} \rightarrow h h$ decay branching ratio $\mathrm{BR}\left(h^{\prime} \rightarrow h h\right)$ is given in terms of the coupling $g_{h^{\prime} h h}$ of Eq. (12). In Tab. 1 the explicit values of the cross section and decay ratio of $h^{\prime}$ are presented for the three values of $m_{h^{\prime}}$, under consideration. For discovery of $h^{\prime}$

\begin{tabular}{|c|c|c|c|}
\hline$m_{h^{\prime}}(\mathrm{GeV})$ & $\sigma\left(p p \rightarrow h^{\prime}\right)(\mathrm{pb})$ & $\mathrm{BR}\left(h^{\prime} \rightarrow h h\right)$ & $\sigma\left(p p \rightarrow h^{\prime} \rightarrow h h \rightarrow b \bar{b} \gamma \gamma\right)(\mathrm{fb})$ \\
\hline 250 & 12.140 & 0.30 & 6.30 \\
\hline 400 & 5.050 & 0.20 & 1.01 \\
\hline 600 & 0.504 & 0.18 & 0.05 \\
\hline
\end{tabular}

TABLE 1: $p p \rightarrow h^{\prime}$ production cross section and its $h^{\prime} \rightarrow h h$ decay branching ratio and the total cross section for its production and decay process $p p \rightarrow h^{\prime} \rightarrow h h \rightarrow$ $b \bar{b} \gamma \gamma$ for three different values of $m_{h^{\prime}}=250 \mathrm{GeV}, 400 \mathrm{GeV}$ and $600 \mathrm{GeV}$.

at the LHC, we analyse signal with the corresponding relevant background from the SM processes. we found following reducible background processes [14]:

$$
p p \rightarrow b b h \gamma \gamma / b b j a / b b j j / c c \gamma \gamma / c c j \gamma / j j \gamma \gamma / g g h \gamma \gamma / t t / t t \gamma / t t h \gamma \gamma / b b z \gamma \gamma / z h \gamma \gamma \text {. }
$$

and we take The following pre-selection cuts at parton level becouse we avoid any divergence in the parton-level calculations [15, 14]:

1. The pseudorapidity $\eta$ of the two photons must be in acceptance of detector so $\left|\eta_{\gamma \gamma}\right| \leq 2.4$.

2. The pseudorapidity $\eta$ of the two jets must be in acceptance of detector so $\left|\eta_{j j}\right| \leq 2.4$.

3. The transverse momentum $P_{T}$ of the two jets satisfies $\left(P_{T}\right)_{j j} \geq 20 \mathrm{GeV}$.

4. The transverse momentum $P_{T}$ of the two photon satisfies $\left(P_{T}\right)_{\gamma \gamma} \geq 25 \mathrm{GeV}$.

All these backgrounds are dead duo to last cuts only relevant background processes alive and compete with our signal are the irreducible ones $p p \rightarrow b \bar{b} \gamma \gamma$. this one cam reduce down by cuts which are used in Tab. 2. In Fig. 3, we show the number of signal events distributions for $m_{h^{\prime}}=250 \mathrm{GeV}$ and $400 \mathrm{GeV}$ with the background before (left) and after (right) cuts in Tab. 2, resepectively.

because the cross section for $m_{h^{\prime}}=600 \mathrm{GeV}$ is quite small so we should go a large luminosity to can make analysis and probe it and this appear in Fig. 4, for $L_{\text {int }}$ values which vary from $100 \mathrm{fb}^{-1}$ up to $3000 \mathrm{fb}^{-1}$ at $\sqrt{s}=14 \mathrm{TeV}$, for the usual three values of $m_{h^{\prime}}$. It is clear that the signal significance increases with considered $L_{\text {int }}$ for each value of $m_{h^{\prime}}$ giving better chances of $h^{\prime}$ discovery with higher $L_{\text {int }}$. 


\begin{tabular}{|c|c|c|c|}
\hline Cuts (Select) & Signal (S): $m_{h^{\prime}}=250 \mathrm{GeV}(400 \mathrm{GeV})$ & Background (B) & $S / \sqrt{\mathrm{B}}$ \\
\hline Initial (no cut) & $1904.00(308.00)$ & 25058.00 & $12.000(1.950)$ \\
\hline$M_{\gamma \gamma}>119.5 \mathrm{GeV}$ & $846.70 \pm 21.70(177.95 \pm 8.82)$ & $3015.10 \pm 51.50$ & $15.419 \pm 0.00527(3.241 \pm 0.00272)$ \\
\hline$M_{\gamma \gamma}<130.5 \mathrm{GeV}$ & $843.90 \pm 19.30(175.80 \pm 8.36)$ & $766.40 \pm 19.20$ & $30.430 \pm 0.01500(6.319 \pm 0.01540)$ \\
\hline
\end{tabular}

TABLE 2: Cut flow charts for the $h^{\prime} \rightarrow h h \rightarrow b \bar{b} \gamma \gamma$ signal versus its relevant background and the corresponding number of events and significance at $300 \mathrm{fb}^{-1}$ and $\sqrt{s}=14 \mathrm{TeV}$ for $m_{h^{\prime}}=250 \mathrm{GeV}(400 \mathrm{GeV})$.

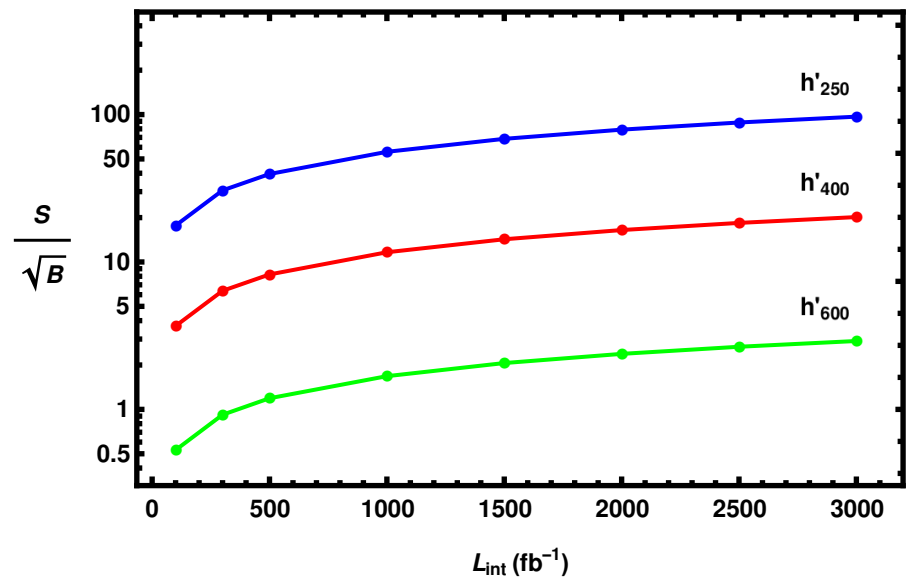

FIGURE 4: Significace of the $h^{\prime} \rightarrow b \bar{b} \gamma \gamma$ signal of Fig. 3 relative to the corresponding background versus $L_{\mathrm{int}}$ at mass $m_{h^{\prime}}=250 \mathrm{GeV}$ (blue), $400 \mathrm{GeV}$ (red) and $600 \mathrm{GeV}$ (green). Data is produced at $\sqrt{\mathrm{s}}=14 \mathrm{TeV}$ and points are interpolated between values of $L_{\text {int }}=100,300,500,1000,1500,2000,2500 \mathrm{fb}^{-1}$ and $L_{\text {int }}=3000 \mathrm{fb}^{-1}$.
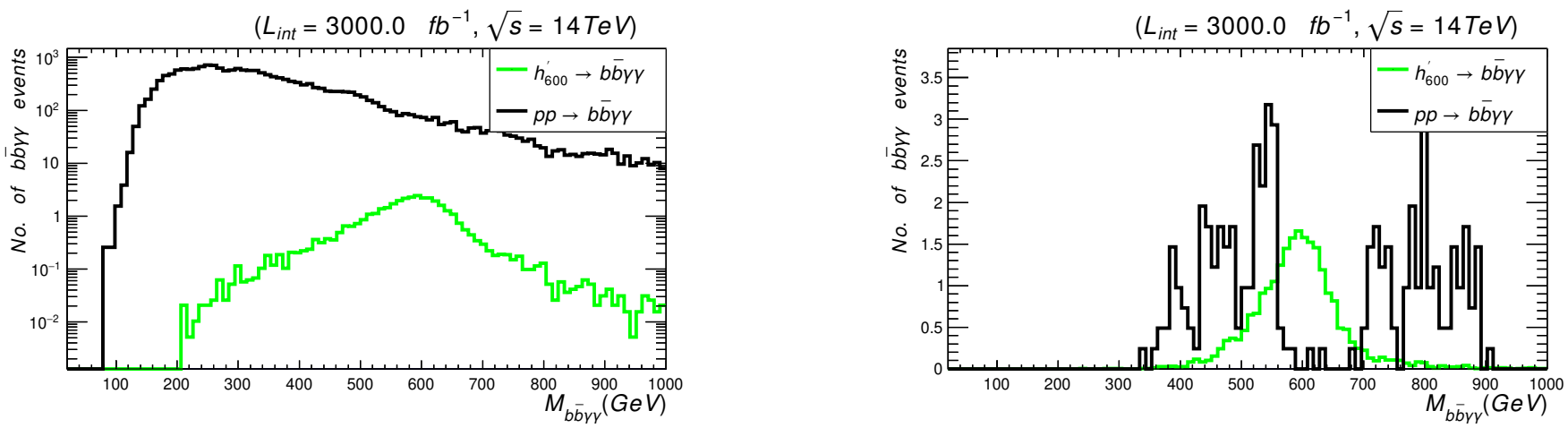

FIGURE 5: Number of signal events for $h^{\prime} \rightarrow b \bar{b} \gamma \gamma$ decays at mass $m_{h^{\prime}}=600 \mathrm{GeV}$ (green) induced by ggF versus the invariant mass of the final states $b \bar{b} \gamma \gamma$, at $\sqrt{s}=14 \mathrm{TeV}$ and $L_{\mathrm{int}}=3000 \mathrm{fb}^{-1}$ alongside the relevant background events background (black) before (left) and after (right) applying the cut flow set of Tab. 3. The corresponding values of cross sections and branching ratios are given in Tab. 1.

With $m_{h^{\prime}}=600 \mathrm{GeV}$, one must go to higher $L_{\mathrm{int}} \sim 3000 \mathrm{fb}^{-1}$, as the the decay and cross section are small. Here we apply the cut flow given in Tab. 3. After all these cuts applying, the final distributions of this event are shown in Fig. 5. According to the plots shown in Fig. 4 and Fig. 5, it is clear that even $h^{\prime}$ with $m_{h^{\prime}} \gtrsim 600 \mathrm{GeV}$ can still be discovered, but at the High-Luminosity Large Hadron Collider (HL-LHC), as it requires $L_{\text {int }}$ of order $L_{\text {int }}=3000 \mathrm{fb}^{-1}$.

In summary, we have proposed a simplified LR model, where $S U(2)_{R} \times U(1)_{B-L}$ symmetry is broken spontaneously by the $v e v$ of a scalar doublet $\chi_{R}$ around TeV scale, and the electroweak symmetry $S U(2)_{L} \times U(1)_{Y}$ is broken by the vevs of two Higgs doublets merged from a single bidoublet $\phi$. We adopted IS mechanism to generate light neutrino masses. We also analysed the Higgs sector in detail, in particular the three neutral CP-even Higgs bosons. We showed that the lightest of these particle can be assigned to SM-like Higgs, with mass equals to $125 \mathrm{GeV}$. The next lightest Higgs, $h^{\prime}$, which is stemmed from the bidoublet neutral component is of order a few hundred GeVs. We studied the LHC potential discovery for $h^{\prime}$ in this class of models. We performed analysis for searches for $h^{\prime}$ by looking for resonant peaks in the following two processes: $h^{\prime} \rightarrow h h \rightarrow b \bar{b} \gamma \gamma$ and $h^{\prime} \rightarrow Z Z \rightarrow 4 \ell(\ell=$ $e, \mu)$. We considered three benchmark points, with $m_{h^{\prime}}=250 \mathrm{GeV}, 400 \mathrm{GeV}$ and $600 \mathrm{GeV}$, at $\sqrt{s}=14 \mathrm{TeV}$ and $L_{\mathrm{int}}=300 \mathrm{fb}{ }^{-1}$ and $L_{\text {int }}=3000 \mathrm{fb}^{-1}$. We emphasized that $h^{\prime}$ can be probed with good statistical significances in di-Higgs channel, with $2 \gamma+2 b$-jets final states. While the channel of $Z$-pair production and decays to $4 \ell$ is much less significant and it may be observed only at very high $L_{\mathrm{int}}=3000 \mathrm{fb}^{-1}$ and for light $h^{\prime}$ with mass less than $300 \mathrm{GeV}$. 


\begin{tabular}{|c|c|c|c|}
\hline Cuts (Select) & Signal (S): $m_{h^{\prime}}=600 \mathrm{GeV}$ & Background (B) & $\mathrm{S} / \sqrt{\mathrm{B}}$ \\
\hline Initial (no cut) & 155.000 & 250650.00 & 0.310 \\
\hline$M_{b b}<200.0 \mathrm{GeV}$ & $52.250 \pm 5.18$ & $39823.60 \pm 82.40$ & $0.264 \pm 0.0008$ \\
\hline$M_{\gamma \gamma}>119.5 \mathrm{GeV}$ & $34.436 \pm 5.91$ & $4252.00 \pm 64.70$ & $0.530 \pm 0.0010$ \\
\hline$M_{\gamma \gamma}<130.5 \mathrm{GeV}$ & $33.542 \pm 5.13$ & $1084.10 \pm 32.00$ & $1.018 \pm 0.0004$ \\
\hline$(\Delta R)_{\gamma \gamma}<2.0$ & $29.230 \pm 4.35$ & $200.50 \pm 17.08$ & $1.062 \pm 0.0500$ \\
\hline$(\Delta R)_{b b}<2.0$ & $27.680 \pm 4.46$ & $132.83 \pm 7.66$ & $2.409 \pm 0.0200$ \\
\hline$\left(P_{T}\right)_{\gamma \gamma}>200.0 \mathrm{GeV}$ & $21.650 \pm 4.36$ & $57.65 \pm 7.70$ & $2.851 \pm 0.0260$ \\
\hline
\end{tabular}

TABLE 3: Cut flow charts for the $h^{\prime} \rightarrow h h \rightarrow b \bar{b} \gamma \gamma$ signal versus its relevant background and the corresponding number of events and significance at $L_{\mathrm{int}}=3000 \mathrm{fb}^{-1}$ and $\sqrt{s}=$ $14 \mathrm{TeV}$ for $m_{h^{\prime}}=600 \mathrm{GeV}$.

\section{References}

[1] G. Senjanovic and Rabindra N. Mohapatra. Exact Left-Right Symmetry and Spontaneous Violation of Parity. Phys.Rev., D12:1502, 1975.

[2] N.G. Deshpande, J.F. Gunion, Boris Kayser, and Fredrick I. Olness. Left-right symmetric electroweak models with triplet Higgs. Phys.Rev., D44:837-858, 1991.

[3] M. Ashry, K. Ezzat, and S. Khalil. Search for Heavy Neutral Higgs Boson in Left-Right Model with Inverse-Seesaw at the LHC. 12021.

[4] R.N. Mohapatra. Mechanism for Understanding Small Neutrino Mass in Superstring Theories. Phys. Rev. Lett., 56:561-563, 1986.

[5] R.N. Mohapatra and J.W.F. Valle. Neutrino Mass and Baryon Number Nonconservation in Superstring Models. Phys. Rev. D, $34: 1642,1986$.

[6] M.C. Gonzalez-Garcia and J.W.F. Valle. Fast Decaying Neutrinos and Observable Flavor Violation in a New Class of Majoron Models. Phys. Lett. B, 216:360-366, 1989.

[7] C. Weiland. Enhanced lepton flavour violation in the supersymmetric inverse seesaw. J. Phys. Conf. Ser., 447:012037, 2013.

[8] Vedran Brdar and Alexei Yu Smirnov. Low Scale Left-Right Symmetry and Naturally Small Neutrino Mass. JHEP, 02:045, 2019.

[9] Georges Aad et al. Combination of searches for Higgs boson pairs in $p p$ collisions at $\sqrt{s}=13 \mathrm{TeV}$ with the ATLAS detector. Phys. Lett. B, 800:135103, 2020.

[10] Albert M Sirunyan et al. Search for nonresonant Higgs boson pair production in final states with two bottom quarks and two photons in proton-proton collisions at $\sqrt{s}=13 \mathrm{TeV}$. JHEP, 03:257, 2021.

[11] Debasish Borah, Sudhanwa Patra, and Utpal Sarkar. TeV scale Left Right Symmetry with spontaneous D-parity breaking. Phys.Rev., D83:035007, 2011.

[12] Georges Aad et al. Observation of a new particle in the search for the Standard Model Higgs boson with the ATLAS detector at the LHC. Phys. Lett. B, 716:1-29, 2012.

[13] Serguei Chatrchyan et al. Observation of a new boson at a mass of $125 \mathrm{GeV}$ with the CMS experiment at the LHC. Phys.Lett., B716:30-61, 2012.

[14] Jung Chang, Kingman Cheung, Jae Sik Lee, Chih-Ting Lu, and Jubin Park. Higgs-boson-pair production $H(\rightarrow b b) H(\rightarrow \gamma \gamma)$ from gluon fusion at the HL-LHC and HL-100 TeV hadron collider. Phys. Rev. D, 100(9):096001, 2019.

[15] The-ATLAS-Collaboration. Study of the double Higgs production channel $H(\rightarrow b \bar{b}) H(\rightarrow \gamma \gamma)$ with the ATLAS experiment at the HL-LHC. ATLAS NOTE, ATL-PHYS-PUB-2017-001(ATL-PHYS-PUB-2017-001), 12017. 